\title{
An IT Platform Enabling Remote Therapeutic Interventions
}

\author{
Marc Schickler ${ }^{1}$, Rüdiger Pryss ${ }^{1}$, Michael Stach ${ }^{1}$, Johannes Schobel ${ }^{1}$, \\ Winfried Schlee ${ }^{2}$, Thomas Probst ${ }^{1}$, Berthold Langguth ${ }^{2}$, Manfred Reichert ${ }^{1}$ \\ ${ }^{1}$ Institute of Databases and Information Systems, Ulm University, Germany \\ ${ }^{2}$ Clinic and Policlinic for Psychiatry and Psychotherapy, University of Regensburg, Germany \\ ${ }^{1}$ \{marc.schickler, ruediger.pryss, michael.stach, johannes.schobel, thomas.probst, manfred.reichert\}@uni-ulm.de \\ winfried.schlee@gmail.com, berthold.langguth@medbo.de
}

\begin{abstract}
The development of information systems, which support homework in the context of therapeutic interventions, has not been sufficiently addressed so far. However, both therapists and patients crave for a mobile assistance managing complex homework procedures. For example, smart mobile devices can automatically inform therapists about corresbonding outcomes, giving them the opportunity to timely adjust homework if required. When realizing information systems that integrate smart mobile devices, the common procedure of therapeutic interventions in general and homework in particular must be carefully captured by the system. Therefore, relevant requirements were elicitated in real-world projects. Based on these requirements, we realized the Albatros platform enabling therapists to manage therapeutic interventions remotely. Using the platform, homework can be created with a web-based component and be performed by patients with the help of smart mobile devices. In this paper, elicitated requirements for realizing the platform as well as its features and architecture are presented. Altogether, the Albatros platform enables therapists as well as patients to manage therapeutic interventions and homework more efficiently.
\end{abstract}

Keywords-Therapeutic intervention, mobile therapy, mobile healthcare, mobile healthcare assistance

\section{INTRODUCTION}

Many studies have confirmed that therapeutic interventions constitute a fundamental part of most therapies as they increase their efficacy. The variety of therapeutic interventions, in turn, ranges from simple medication intakes up to complex behavioral therapies including homework, which is assigned by a therapist to the patient. In particular, homework supports therapeutic progress and helps to transfer therapeutic achievements from the clinical setting to the real-life situation of the patient at home. Taking this diversity into account, many therapists crave for a more sophisticated IT support. In line with this trend, recent studies (e.g., [1]) revealed that smart mobile devices are demanded by therapists and can be used to obtain new insights in clinical research. However, existing approaches still come with several drawbacks. First, they mainly provide specifically tailored applications and often do not cope with the diversity of therapeutic interventions. Second, they neither consider the execution context of homework properly nor the provision of feedback. Third, existing solutions focus on the therapist-patient relationship and, therefore,

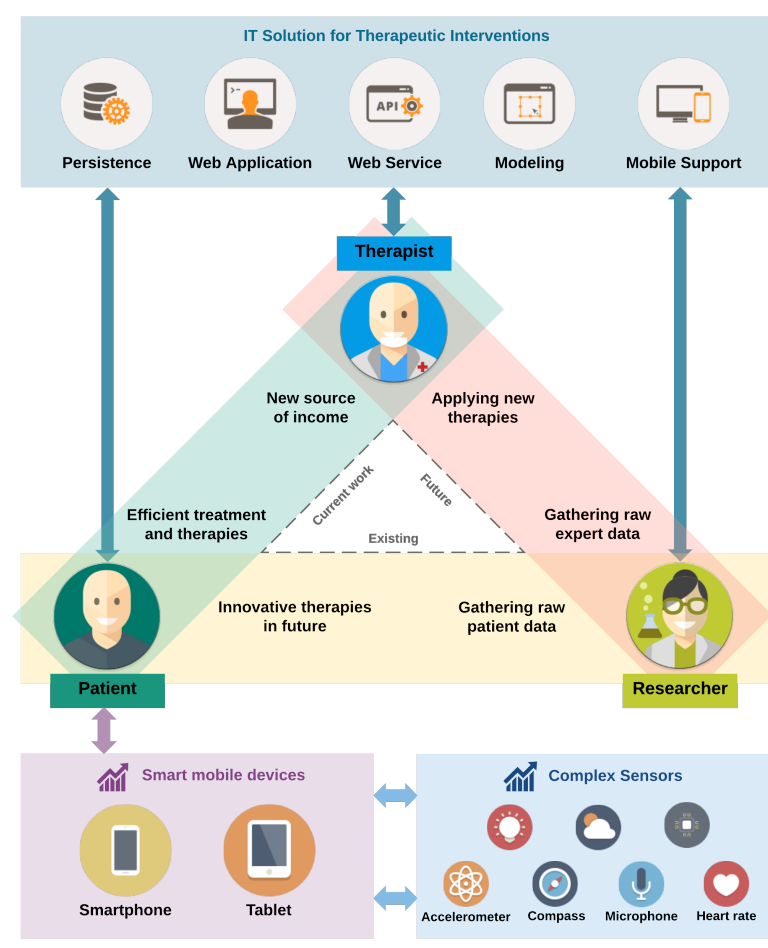

Figure 1: IT Platform Challenges

neglect emerging opportunities (e.g., context-sensitive data) in this context. Fourth, features provided by current smart mobile devices (e.g., sensors) have seldomly been used for therapeutic interventions.

Taking these shortcomings into account, Fig. 1 illustrates the three major challenges addressed by the Albatros platform. First, smart mobile devices need to be integrated. Second, the capabilities offered by smart mobile device sensors shall be utilized. Third, the therapist-patient relationship shall be extended to the therapist-patient-researcher relationship. For this purpose, features must be provided that enable the three stakeholders to collaborate. For example, as therapists manage their individually created therapeutic interventions digitally when using the Albatros platform, they can easily make them available for researchers. As 
a consequence, the therapist-researcher relationship can be enhanced since researchers may evaluate the efficacy of therapeutic interventions based on proper longitudinal data. From a technical perspective, four aspects are crucial when realizing the Albatros platform:

1) Insights into real-world therapies as well as a literature study are required to understand the common procedure for managing therapeutic interventions.

2) The shortcomings of the identified procedure shall be analyzed to elicitate requirements for the Albatros platform.

3) These requirements, in turn, shall be addressed to help evolve existing procedures for therapeutic interventions. In particular, the contextual information as well as the provision of feedback shall be included.

4) The Albatros architecture shall support the optimized procedure to efficiently perform therapeutic interventions.

The remainder of the paper is structured as follows: Section II discusses the common procedure for managing therapeutic interventions as well as its current shortcomings and elicitated requirements. Section III presents an optimized procedure for the support of therapeutic interventions, which meets these requirements. Section IV presents the Albatros architecture, whereas Section V introduces the implemented prototype. Section VI discusses related work and Section VII concludes the paper with a summary.

\section{Common Therapeutic Intervention Procedure}

The development of an IT platform supporting remote therapeutic interventions necessitates insights into real-world procedures. These insights were gathered by us through an analysis of six scenarios from the real world; i.e., we investigated six large-scale solutions supporting therapeutic interventions with specifically tailored applications (cf. Table I). This revealed that an appropriate IT support significantly increases the efficacy of therapeutic interventions. For example, in psychosomatic in-patient clinics, clinical support tools increased the outcome for patients.

Fig. 2 shows how therapeutic interventions are usually accomplished in the considered real-world scenarios. As the

\begin{tabular}{l|l|c|r}
\hline Approach & Country & \#STI & Patient Cases \\
\hline \hline TrackYourTinnitus [2] & World-Wide & 12 & 1,500 \\
Tinnitus Hearing Ability [3] & Germany & 10 & 120 \\
Risk Factors during & Germany & 12 & 410 \\
Pregnancy [4] & & & 560 \\
PTSD in War Regions [5] & Burundi & 6 & 80 \\
Supporting Parents after & Switzerland, & 6 & 25 \\
Accidents of Children [6] & Germany & & \\
Psychosomatic & Germany & 23 & 2,870 \\
in-patients clinics [7], [8] & & 59 & \\
\hline \hline Sum $\Sigma$ & &
\end{tabular}

Table I: Approaches Supporting Therapeutic Interventions

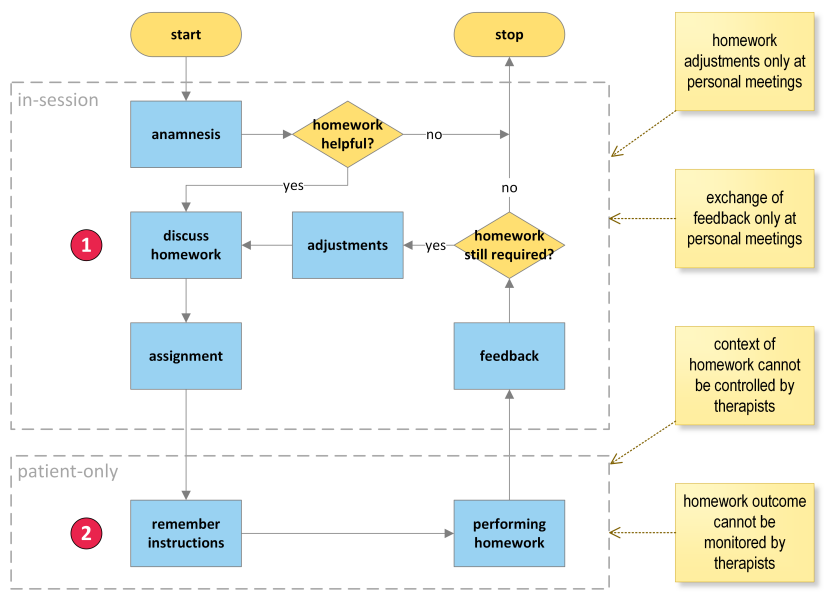

Figure 2: Common Therapeutic Intervention Procedure

depicted procedure is crucial for the design of the Albatros platform, we summarize it in the following: the therapy starts with a first personal meeting of the therapist and the patient. During this meeting as well as the follow-up sessions, the therapist creates a comprehensive anamnesis for the case conceptualization, based on which the therapeutic interventions (e.g., homework) are planned. If homework becomes necessary, the therapist discusses it with the patient and specifies exercises (e.g., relaxation techniques) that need to be performed to successfully complete the homework (cf. Fig. 2). In this context, exercises must be explained to the patient (i.e., what, why, when, where, how, how often, with whom). If homework is not explained to the patient in detail, he might forget it in daily life or, especially when performing complex exercises (e.g., cognitive restructuring). If exercises are not performed as intended by therapists their efficacy is potentially mitigated. Other shortcomings of current manual therapeutic intervention procedures are as follows:

- Homework adjustments are only performed in personal meetings of the therapist and the patient. Hence, adjustments might be ineffective.

- Homework cannot be monitored efficiently and, therefore, therapists are unable to adjust ongoing homework timely.

- Patients have difficulties in providing detailed feedback on the performed homework. Even if they have not, the feedback might be biased, as sharing the experiences with the therapist is only possible retrospectively at personal meetings.

- Therapists cannot control the context in which homework is performed. If patients do not stick to a helpful context, homework efficacy will be low.

- As crucial parts (e.g., complex exercises) of therapeutic intervention procedures are not computerized, the reuse of already existing homework is difficult.

- Therapists are unable to share homework schemes with 
other therapists in a convenient manner. Accordingly, the exchange of homework schemes within therapeutic institutions is limited.

Considering these shortcomings, we elicitated technical requirements to be met by the Albatros platform (cf. Table II). We divide the requirements into four categories: (1) Requirements related to exercises; e.g., therapists shall be able to assign media elements to exercises, which are then presented to patients on their smart mobile devices; (2) requirements related to the context of an exercise. This context shall enable the Albatros platform to cope with the demands of therapists on one hand (e.g., a homework may have to be performed after getting up) and to provide a powerful tool for researchers to gather valuable contextsensitive data on the other; (3) requirements related to patient notifications; e.g., a patient shall be automatically notified to perform the assigned homework; (4) requirements related to feedback; e.g., therapists shall be able to create a feedback schema using different question types. Altogether, 13 requirements were identified, which shall be met by the Albatros platform.

\section{IT SUPPORTED INTERVENTION PROCEDURE}

The development of the Albatros platform takes two major aspects into account. The (1) technical requirements shown in Table II are considered and (2) therapists are enabled to exchange homework schemes with other therapists. To properly address these two issues, the procedure shown in Fig. 3 was developed.

Regarding exercises, four aspects become relevant:

(1) Exercises are stored in a repository (cf. Fig. 3, (1)). This enables therapists of the same institute to share exercises. Note that the Albatros platform also supports the exchange of exercises between healthcare organizations, which is not further considered in this paper.

\begin{tabular}{lll}
\hline No & Title & Description \\
\hline Category I: Exercise & \\
1 & Configuration & Configure exercise (e.g., description) \\
2 & Versioning & Create and manage exercise versions \\
3 & Reuse & Use exercise for different scenarios \\
4 & Media & Use media elements for exercise (e.g., videos) \\
\hline Category II: Context & \\
5 & Configuration & Configure context (e.g., date, location) \\
6 & Versioning & Create and manage context versions \\
7 & Reuse & Use context for different scenarios \\
8 & Sensors & Utilize sensors of smart mobile devices \\
\hline Category III: Notification \\
9 & Schema & Create context-sensitive notification schema \\
10 & Patient & Notify patient to perform exercise \\
\hline Category IV: & Feedback \\
11 & Schema & Create feedback schema \\
12 & Media & Upload media elements (e.g., videos, pictures) \\
13 & Reuse & Use feedback schema for different scenarios \\
\hline
\end{tabular}

Table II: IT Platform Requirements
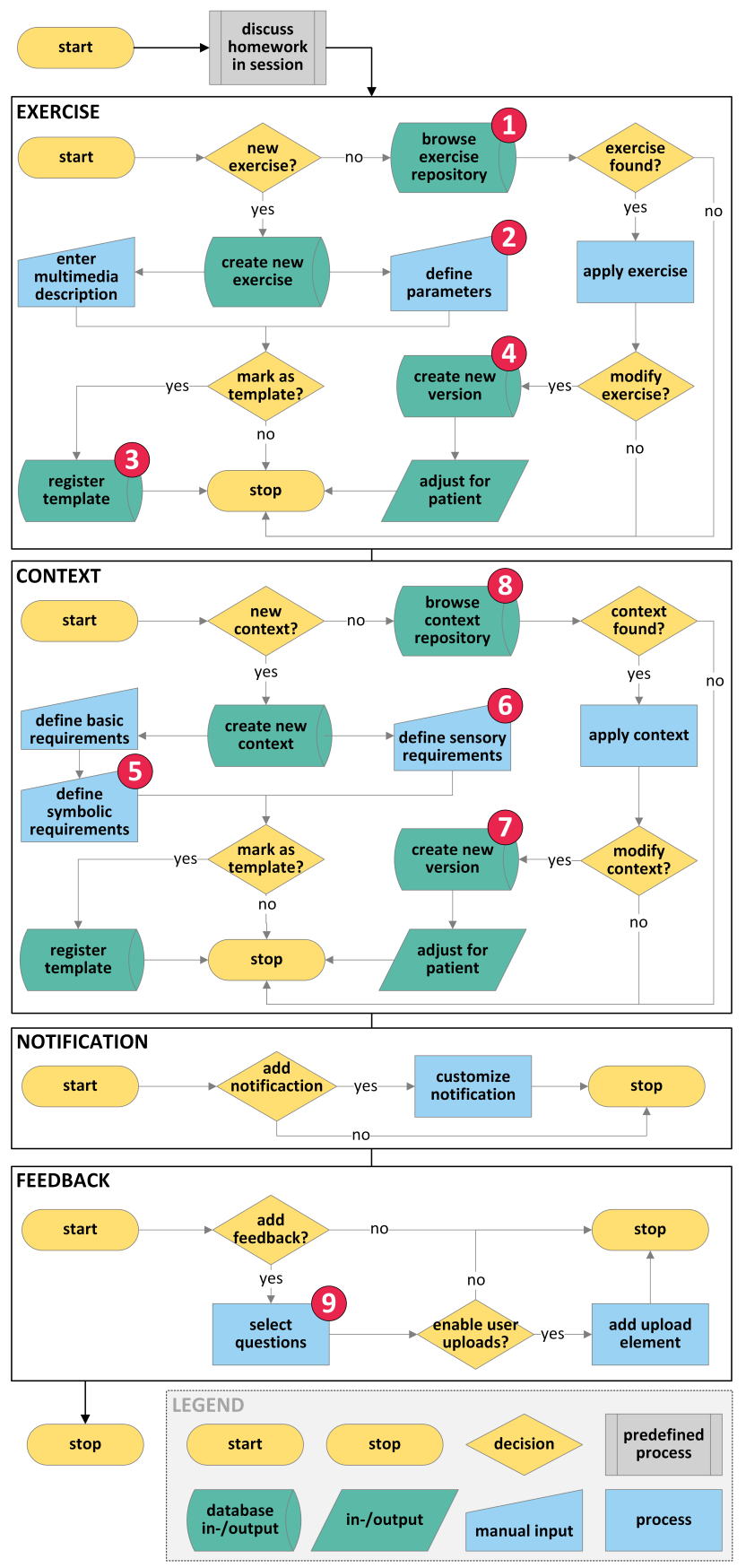

Figure 3: IT Supported Therapeutic Intervention Procedure

(2) A set of parameters (e.g., number of repetitions, duration) is provided in order to cope with the variety of exercises (cf. Fig. 3, (2)). This parameter set can be easily extended.

(3) To ease the reuse of exercises, a template feature was developed (cf. Fig. 3, (3)); e.g., a template for a breathing exercise, for which only the number of repetitions needs to be set, may be defined.

(4) During most therapies, exercises need to be adjusted 
to increase their efficacy over time. Therapists want to track respective adjustments, necessitating the support of multiple versions of an exercise (cf. Fig. 3, (4)).

Regarding the context, in which exercises are performed, the following three aspects become relevant:

(1) For specifying the context, two kinds of parameters are provided. Symbolic parameters (cf. Fig. 3, (5)) describe facts to be ensured by the patients themselves (e.g., doing a breathing exercise in the living room, which represents the symbolic parameter). In turn, sensory parameters (cf. Fig. 3, (6) are automatically evaluated by the smart mobile devices of the patients (e.g., measuring the heart rate, which is considered as sensory parameter).

(2) A template as well as a versioning concept (cf. Fig. 3 , (7) is provided for capturing contextual schemes.

(3) Context specifications (i.e., context schemes) shall be reusable. In turn, this requires a context repository (cf. Fig. 3, (8)).

Concerning the feature that enables patients to provide feedback to their therapists, two aspects are important:

(1) For each exercise, a feedback questionnaire can be defined (cf. Fig. 3, (9)). The latter consists of different question types (e.g., single and multiple choice, Likert scale).

(2) Patients may enrich feedback by uploading additional elements (e.g., videos or spreadsheets).

Concerning the notification feature, a platform-specific notification is automatically triggered based on the context specified by therapists. Furthermore, therapists may customize the user interface template of a notification (e.g., default color, font).

\section{Albatros Architecture}

Fig. 4 illustrates the Albatros architecture, which enables the realization of the discussed therapeutic intervention procedure. Although the concept is not restricted to specific technologies, we realized the prototype using the Microsoft .NET Framework and SQL Server. In turn, the mobile application supporting patients with therapeutic interventions at home is realized using Android.

Taking the elaborated requirements into account, several aspects of the architecture need to be discussed. First, as many stakeholders are involved, a robust access control is required. For this purpose, we implemented a sophisticated authorization \& authentication service (cf. Fig. 4 (1)), taking contemporary security and data protection concepts into account. Second, as an additional demand of therapists, the invitation service (cf. Fig. 4 (2)) was implemented. To be more precise, a therapist must be able to invite patients by using an anonymized and unique invitation code. The latter can be easily created by the Albatros platform. Third, we implemented a notification service (cf. Fig. 4 (3)) being

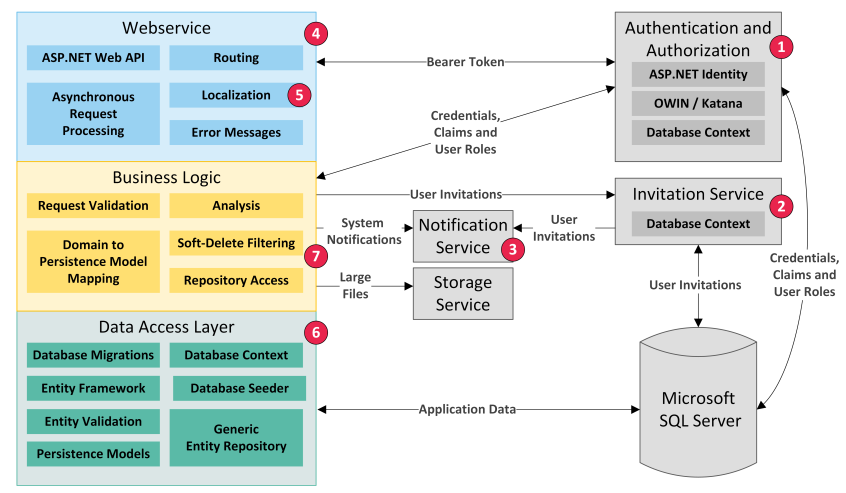

Figure 4: Albatros Architecture

able to send e-mails to patients and push notifications to the patients' smart mobile devices. Fourth, we implemented a RESTful web service (cf. Fig. 4 (4)) to manage the entire communication with the smart mobile devices. In addition, the communication required for the HTML5 application used by therapist is managed through the web service as well. In this context, the web service offers features for multilingualism (cf. localization web service component shown in Fig. 4 (5)). Fifth, we designed a feasible data model (cf. Fig. 4 (6) to cope with the relationships between therapeutic interventions, homework, exercises, context, feedback, and notifications. Subsequently, we implemented an advanced business logic (cf. Fig. 4 (7)) and a data access layer that incorporates the defined data model.

\section{PROOF-OF-CONCEPT PROTOTYPE}

We implemented a proof-of-concept prototype, which is briefly discussed in the following. Inspired by the insights from the QuestionSys project and its modeling component [9]-[11], fundamental components of the presented prototype were developed. Currently, the prototype consists of an HTML5 application and a mobile application for the Android mobile operating system. The HTML5 application, in turn, provides the modeling component as well as the component to manage therapeutic interventions by therapists. To provide an impression of the prototype, Fig. 5 shows the therapists' view to manage patient cases. More precisely, all therapeutic interventions, the personal appointments, and the therapy history are managed using this view. In turn, Fig. 6 depicts the view in which therapists may configure all possible homework features, e.g., required media elements for performing a homework. Finally, Fig. 7 shows the list of already created context schemes as well as details of one context schema.

Two issues are finally mentioned that emerged when using the Albatros platform in practice. First, the therapeutic intervention procedure as well as the identified requirements are sufficiently considered by the realized platform. Second, the resulting complexity for therapists to create and manage 


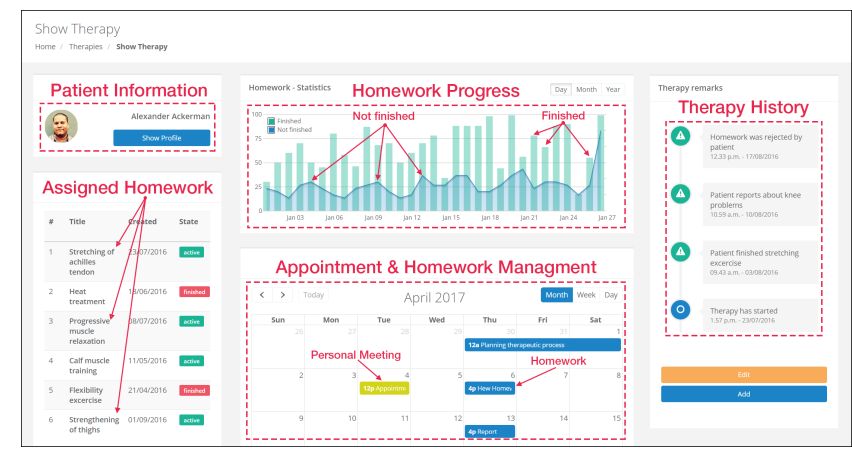

Figure 5: Therapy Details

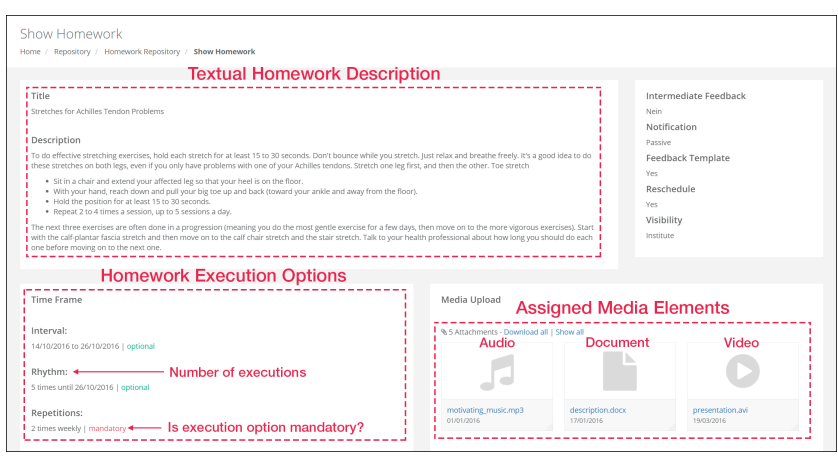

Figure 6: Homework Details

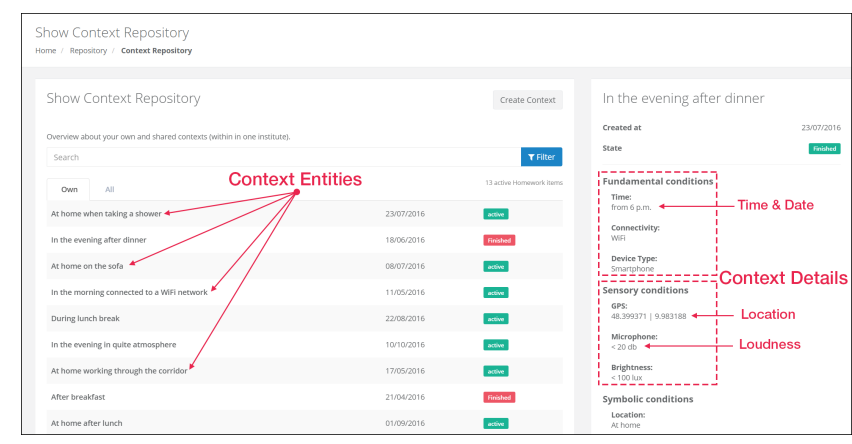

Figure 7: Context Repository

therapeutic interventions is still challenging for them. In this context, insights from the QuestionSys project help to reduce the overall configuration complexity through the use of concepts known from end-user development. Therefore, a large-scale experiment is currently conducted with the goal to identifiy the factors that may reduce the complexity.

\section{RELATED WORK}

Three categories of related work are relevant in the context of this paper.

Approaches providing IT platforms for therapeutic interventions. Online-supported therapeutic interventions and evaluations of the efficacy of respective interventions compared to traditional ones are presented in [12]-[16]. However, only few approaches providing IT support for thera- peutic interventions can be found. Commercial software solutions dealing with e-health and Internet-based therapeutic interventions, in turn, are discussed in [17], [18]. None of these solutions provides support as the Albatros platform does.

Approaches dealing with mobile therapies. Several studies that use mobile phones as a motivating factor for teenagers to record their mental health symptoms are presented in [19]. In this context, recordings are discussed with clinicians. In turn, [20] presents approaches using smartphones in the context of personal healthcare. Recent works, e.g., [21], revealed that mobile applications can be used to properly perform therapeutic interventions. In addition, there exist IT-driven approaches that may be configured by domain experts to increase the efficacy of psychotherapy [22]. In line with [20], [1] elaborates the benefits of mobile technology with respect to the efficacy of psychotherapy in detail. However, none of these approaches presents technical solutions for therapeutic interventions, as the Albatros platform does. [23] presents three clinical studies in which mobile technology was used for mobile interventions. For each study, a specifically tailored mobile application was realized. Although [23] emphasizes the usefulness of mobile interventions in the considered case studies, no generic technical solution is presented.

Approaches dealing with homework in the context of psychotherapy. Numerous works deal with homework in the context of psychotherapy [19], [24], [25]. However, respective approaches mainly address the results of the conducted studies with no particular focus on technical issues. Moreover, no technical solution is presented that applies contemporary mobile technology. In turn, all studies reveal that homework should be a fundamental component of most psychotherapies.

Altogether, existing work has proven that homework is crucial for many therapies. The development of a generic technical solution that supports homework in particular and therapeutic interventions in general is currently less considered.

\section{SUMmARY AND OUTLOOK}

This paper presented an IT platform enabling therapists to provide therapeutic interventions to their patients with the help of smart mobile devices. We discussed fundamental requirements elaborated in real-world projects to capture the common therapeutic intervention procedure with the Albatros platform. Thereby, four major aspects are particularly considered: (1) the creation of exercises, (2) the specification of a context, (3) the provision of a feedback feature, and (4) a notification feature. In this context, we showed the developed procedure that combines these four aspects, the developed architecture as well as the implementation of the prototype. Overall, an approach supporting therapeutic interventions with the use of smart mobile devices becomes increasingly 
important. In current work, we focus on the modeling component, which we evaluate through an experiment. In addition, we will consider legal aspects of the platform. Altogether, the support of therapeutic interventions on smart mobile devices constitutes an important step towards more effective therapies.

\section{REFERENCES}

[1] O. Lindhiem, C. Bennett, D. Rosen, and J. Silk, "Mobile technology boosts the effectiveness of psychotherapy and behavioral interventions a meta-analysis," Behavior modification, vol. 39, no. 6, pp. 785-804, 2015.

[2] R. Pryss, M. Reichert, B. Langguth, and W. Schlee, "Mobile Crowd Sensing Services for Tinnitus Assessment, Therapy and Research," in IEEE 4th Int'l Conf on Mobile Services. IEEE Computer Society Press, 2015.

[3] M. Schickler, R. Pryss, M. Reichert, J. Schobel, B. Langguth, and W. Schlee, "Using Mobile Serious Games in the Context of Chronic Disorders - A Mobile Game Concept for the Treatment of Tinnitus," in 29th IEEE Int'l Symp on ComputerBased Medical Systems, 2016, pp. 343-348.

[4] M. Ruf-Leuschner, N. Brunnemann, M. Schauer, R. Pryss, E. Barnewitz, M. Liebrecht, W. Kratzer, M. Reichert, and T. Elbert, "The KINDEX-App - an Instrument for Assessment and Immediate Evaluation of Psychosocial Risk Factors in Pregnant Women in Daily Practice by Gynecologists, Midwives and in Gynecological Hospitals," Verhaltenstherapie, vol. 26, no. 3, pp. 171-181, 2016.

[5] J. Schobel, R. Pryss, and M. Reichert, "Using Smart Mobile Devices for Collecting Structured Data in Clinical Trials: Results From a Large-Scale Case Study," in 28th IEEE Int'l Symposium on Computer-Based Medical Systems. IEEE Computer Society Press, 2015.

[6] "Kidtrauma," https://kidtrauma.com/en/, 2017, [Online; accessed 19-April-2017].

[7] T. Probst, M. Lambert, T. Loew, R. Dahlbender, R. Göllner, and K. Tritt, "Feedback on patient progress and clinical support tools for therapists: Improved outcome for patients at risk of treatment failure in psychosomatic in-patient therapy under the conditions of routine practice," Journal of Psychosomatic Research, vol. 75, no. 3, pp. 255-261, 2013.

[8] T. Probst, M. Lambert, R. Dahlbender, T. Loew, and K. Tritt, "Providing patient progress feedback and clinical support tools to therapists: Is the therapeutic process of patients on-track to recovery enhanced in psychosomatic in-patient therapy under the conditions of routine practice?" Journal of psychosomatic research, vol. 76, no. 6, pp. 477-484, 2014.

[9] J. Schobel, R. Pryss, W. Wipp, M. Schickler, and M. Reichert, "A Mobile Service Engine Enabling Complex Data Collection Applications," in 14th Int'l Conf on Service Oriented Computing, ser. LNCS, 2016, pp. 626-633.

[10] J. Schobel, R. Pryss, M. Schickler, M. Ruf-Leuschner, T. Elbert, and M. Reichert, "End-User Programming of Mobile Services: Empowering Domain Experts to Implement Mobile Data Collection Applications," in 5th IEEE Int'l Conf on Mobile Services, 2016, pp. 1-8.
[11] J. Schobel, R. Pryss, M. Schickler, and M. Reichert, "A Configurator Component for End-User Defined Mobile Data Collection Processes," in Proc 14th Int'l Conf on Service Oriented Computing, October 2016.

[12] A. Barak, B. Klein, and J. Proudfoot, "Defining internetsupported therapeutic interventions," Annals of Behavioral Medicine, vol. 38, no. 1, pp. 4-17, 2009.

[13] V. Spek, P. Cuijpers, I. Nyklícek, H. Riper, J. Keyzer, and V. Pop, "Internet-based cognitive behaviour therapy for symptoms of depression and anxiety: a meta-analysis," Psychological medicine, vol. 37, no. 03, pp. 319-328, 2007.

[14] S. Walters, J. Wright, and R. Shegog, "A review of computer and internet-based interventions for smoking behavior," $A d$ dictive behaviors, vol. 31, no. 2, pp. 264-277, 2006.

[15] A. Barak, L. Hen, M. Boniel-Nissim, and N. Shapira, "A comprehensive review and a meta-analysis of the effectiveness of internet-based psychotherapeutic interventions," Journal of Technology in Human services, vol. 26, no. 2-4, pp. 109-160, 2008.

[16] M. Lambert, "Progress feedback and the OQ-system: The past and the future." Psychotherapy, vol. 52, no. 4, p. 381, 2015.

[17] "Minddistrict," https://www.minddistrict.com, 2017, [Online; accessed 19-April-2017].

[18] E. AbuKhousa, N. Mohamed, and J. Al-Jaroodi, "e-health cloud: opportunities and challenges," Future Internet, vol. 4, no. 3, pp. 621-645, 2012.

[19] D. J. Cox, D. A. Tisdelle, and J. Culbert, "Increasing adherence to behavioral homework assignments," Journal of behavioral medicine, vol. 11, no. 5, pp. 519-522, 1988.

[20] K. Wac, "Smartphone as a personal, pervasive health informatics services platform: literature review," arXiv preprint arXiv:1310.7965, 2013.

[21] A. Aguilera and F. Muench, "There's an app for that: Information technology applications for cognitive behavioral practitioners," The Behavior therapist/AABT, vol. 35, no. 4, p. $65,2012$.

[22] S. Watts, A. Mackenzie, C. Thomas, A. Griskaitis, L. Mewton, A. Williams, and G. Andrews, "Cbt for depression: a pilot rct comparing mobile phone vs. computer," $B M C$ psychiatry, vol. 13, no. 1, p. 49, 2013.

[23] C. Depp, B. Mausbach, E. Granholm, V. Cardenas, D. Ben-Zeev, T. Patterson, B. Lebowitz, and D. Jeste, "Mobile interventions for severe mental illness: design and preliminary data from three approaches," The Journal of nervous and mental disease, vol. 198, no. 10, p. 715, 2010.

[24] N. Kazantzis, C. Whittington, and F. Dattilio, "Meta-analysis of homework effects in cognitive and behavioral therapy: A replication and extension," Clinical Psychology: Science and Practice, vol. 17, no. 2, pp. 144-156, 2010.

[25] T. Neben, A. Seeger, T. Kramer, and A. White, "Regaining Joy of Life: Theory-Driven Development of Mobile Psychotherapy Support Systems," 2015. 